\title{
Observation of perfect diamagnetism and interfacial effect on the electronic structures in infinite layer

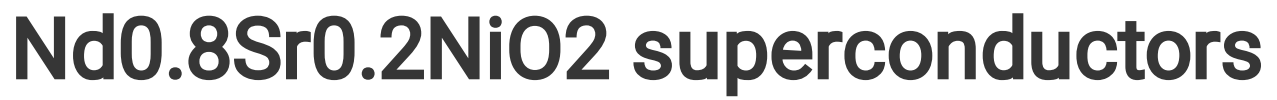

\section{Shengwei Zeng}

National University of Singapore

Xinmao Yin

Shanghai University https://orcid.org/0000-0002-8246-4444

C. J. Li

National University of Singapore

Chi Sin Tang

National University of Singapore

K. Han

National University of Singapore

\section{Z. Huang}

National University of Singapore

Y. Cao

National University of Singapore

\section{E. Chow}

National University of Singapore

D.Y. Wan

National University of Singapore https://orcid.org/0000-0001-6810-2183

\section{Z. T. Zhang}

National University of Singapore

\section{Z. S. Lim}

National University of Singapore https://orcid.org/0000-0002-1034-1234

\section{Caozheng Diao}

Singapore Synchrotron Light Source, National University of Singapore, 5 Research Link, 117603,

Singapore https://orcid.org/0000-0002-6574-9085

\section{Ping Yang}

National University of Singapore

\section{Andrew Wee}

National University of Singapore https://orcid.org/0000-0002-5828-4312

\section{Stephen Pennycook}

National University of Singapore https://orcid.org/0000-0002-3210-6323 


\section{A. Ariando ( $\sim$ phyarian@nus.edu.sg )}

National University of Singapore https://orcid.org/0000-0002-0598-426X

\section{Article}

Keywords: superconductors, condensed-matter physics, materials physics

Posted Date: June 14th, 2021

DOl: https://doi.org/10.21203/rs.3.rs-576278/v1

License: (c) (1) This work is licensed under a Creative Commons Attribution 4.0 International License. Read Full License

Version of Record: A version of this preprint was published at Nature Communications on February 8th, 2022. See the published version at https://doi.org/10.1038/s41467-022-28390-w. 


\section{Observation of perfect diamagnetism and interfacial effect on the}

electronic structures in infinite layer $\mathrm{Ndo.8Sro.2}_{2} \mathrm{NiO}_{2}$ superconductors

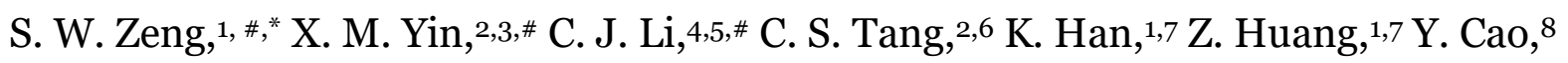
L. E. Chow, ${ }^{1}$ D. Y. Wan, ${ }^{1}$ Z. T. Zhang, ${ }^{1}$ Z. S. Lim,${ }^{1}$ C. Z. Diao, ${ }^{2}$ P. Yang,,${ }^{2,4}$

A. T. S. Wee, ${ }^{1,2,9}$ S. J. Pennycook 4 , A. Ariando, ${ }^{1,9, *}$

${ }^{1}$ Department of Physics, Faculty of Science, National University of Singapore, Singapore 117551, Singapore

2Singapore Synchrotron Light Source (SSLS), National University of Singapore, 5 Research Link, Singapore 117603, Singapore 3Shanghai Key Laboratory of High Temperature Superconductors, Physics Department, Shanghai University, Shanghai 200444, China 4 Department of Materials Science and Engineering, National University of Singapore, Singapore 117575, Singapore

5Department of Materials Science and Engineering, Southern University of Science and Technology, Shenzhen 518055, Guangdong, China

${ }^{6}$ Institute of Materials Research and Engineering, A*STAR (Agency for Science, Technology and Research), 2 Fusionopolis Way, Singapore, 138634 Singapore

7Information Materials and Intelligent Sensing Laboratory of Anhui Province, Institutes of Physical Science and Information Technology, Anhui University, Hefei 230601, Anhui, China

${ }^{8}$ Department of Electrical and Computer Engineering, National University of Singapore, Singapore 117583, Singapore

${ }^{9}$ Centre for Advanced 2D Materials, National University of Singapore, Singapore 117546, Singapore

\#The authors contributed equally to this work.

*To whom correspondence should be addressed: phyzen@nus.edu.sg, ariando@nus.edu.sg 
Nickel-based complex oxides have served as a playground for decades in the quest for a copper-oxide analog of the high-temperature (high-Tc) superconductivity. They may provide key points towards understanding the mechanism and an alternative route for $h i g h-T_{c}$ superconductors. The recent discovery of superconductivity in the infinite-layer nickelate thin films has fulfilled this pursuit ${ }^{1}$. Thus far, however, material synthesis remains challenging. The demonstration of perfect diamagnetism is still missing, and understanding of the role of the interface and bulk to the superconducting properties is still lacking ${ }^{2-16}$. Here, we synthesized high-

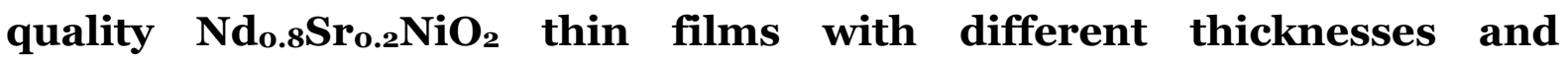
investigated the interface and strain effects on the electrical, magnetic and optical properties. Perfect diamagnetism is demonstrated, confirming the occurrence of superconductivity in the thin films. Unlike the thick films in which the normal-state Hall coefficient $\left(R_{H}\right)$ changes signs as the temperature decreases, the $R_{H}$ of films thinner than $6.1 \mathrm{~nm}$ remains negative, suggesting a thickness-driven band structure modification. Moreover, X-ray absorption spectroscopy reveals the Ni-O hybridization nature in doped infinite-layer nickelates, and the hybridization is enhanced as the thickness decreases. Consistent with band structure calculations on the nickelate/SrTiO ${ }_{3}$ heterostructure, the interface and strain effect induce a dominating electron-like band in the ultrathin film, thus causing the sign change of the $R_{\mathrm{H}}$. 
The search for the nickelate superconductivity was enthused by the idea of mimicking the $3 d_{x^{2}-y^{2}}$ orbital of the single-band high- $T_{\mathrm{c}}$ cuprates $^{1}$. However, the recent results indicate the complex multiband structures in doped infinite-layer nickelates, suggesting a new family of superconductivity ${ }^{1,2}$. Numerous theoretical works have been conducted based on the bulk pictures ${ }^{2}$; however, the superconductivity has only been observed in epitaxial $\mathrm{Nd}_{1-\mathrm{x}} \mathrm{Sr}_{\mathrm{x}} \mathrm{NiO}_{2}$ and $\mathrm{Pr}_{1-\mathrm{x}} \mathrm{Sr}_{\mathrm{x}} \mathrm{NiO}_{2}$ ultrathin films (up to $\sim 10$ $\mathrm{nm}$ ) with an infinite-layer structure ${ }^{1,3-9}$. In contrast, infinite-layer nickelates prepared in bulk form show only insulating behavior ${ }^{10-12}$. Moreover, the DC diamagnetic response in the superconducting thin films has never been demonstrated ${ }^{1}$. These beg the question of whether the superconductivity occurs in the whole film or at the interface between the nickelate and $\mathrm{SrTiO}_{3}$ (STO) substrate ${ }^{13-16}$. Theoretical calculation further proposed that the interface/surface-induced Fermi surface modification causes the transformation from a $d$-wave paring in bulk into an $s$-wave paring at the interface/surface ${ }^{2,17}$, which might be consistent with the recent observation of two gaps from the tunneling spectrum measurement5. This further adds to the puzzle of whether the observed electronic properties are associated with the bulk or the heterostructure interface. Here, we comprehensively investigate the $\mathrm{Nd}_{0.8} \mathrm{Sr}_{0.2} \mathrm{NiO}_{2}$ films of various thicknesses to confirm the bulk nature of the superconductivity and reveal the interfacial effects on the multiband picture of the infinite-layer nickelate thin films and demonstrate their perfect DC diamagnetic response.

Figure 1 (a) shows the X-ray diffraction (XRD) $\theta-2 \theta$ patterns of the $\mathrm{Nd}_{0.8} \mathrm{Sr}_{0.2} \mathrm{NiO}_{2}$ thin films of different thicknesses from 5.1 to $17 \mathrm{~nm}$. The XRD characterization of the asgrown perovskite $\mathrm{Nd}_{0.8} \mathrm{Sr}_{0.2} \mathrm{NiO}_{3}$ thin films can be found in Supplementary Data Fig. S1. The obvious diffraction peak and thickness oscillations in the vicinity of the (00l) 
infinite-layer peak ( $l$ is an integer) indicate the high quality of the films. The $(00 l)$ peak positions slightly shift towards a higher angle as the thickness increases, indicating a shrinking of the $c$-axis, with the lattice constants $d$ change from $\sim 3.42 \AA$ for the 5.1$\mathrm{nm}$ film to $\sim 3.36 \AA$ for the 17-nm film, as plotted in Fig. 1(b). It has been shown that $\mathrm{Nd}_{1-\mathrm{x}} \mathrm{Sr}_{\mathrm{x}} \mathrm{NiO}_{2}(x \leq 0.2)$ bulks exhibit an in-plane lattice constant $(3.914-3.921 \AA$ A $)$ slightly larger than that $(3.905 \AA)$ of STO substrate ${ }^{10,11}$, suggesting the presence of compressive strain on the films imposed by the substrate. The extent of compressive strain decreases as the film thickness increases and thus causing the shrinking of $d$. Figure 1(c) shows the high-angle annular dark-field scanning transmission electron microscopy (HAADF-STEM) image of the $11.3-\mathrm{nm}$ film. A clear infinite-layer structure is observed with no obvious defect throughout the layer.

The resistivity versus temperature $(\rho-T)$ curves for the $\mathrm{Nd}_{0.8} \mathrm{Sr}_{0.2} \mathrm{NiO}_{2}$ thin films are shown in Fig. 2(a), and the zoomed-in $\rho$ - $T$ curves at temperatures from 50 to $2 \mathrm{~K}$ are shown in the inset. All the samples behave like a metal at the normal state and are superconducting at low temperatures. The onset superconducting transition temperature $T_{\mathrm{c}, 90 \% R}$ (defined as the temperature at which the resistivity drops to $90 \%$ of the value at $15 \mathrm{~K}$ ) and zero-resistance $T_{\mathrm{c}, \text { zero- } R}$ decrease with decreasing thickness (Fig. 2(f)). Figure 2(b) shows the corresponding temperature dependence of the normal-state Hall coefficients $R_{\mathrm{H}}$ of the $\mathrm{Nd}_{0.8} \mathrm{Sr}_{0.2} \mathrm{NiO}_{2}$ films. The $R_{\mathrm{H}}$ for samples with a thickness higher than $6.8 \mathrm{~nm}$ shows a negative sign at room temperature and undergoes a smooth transition to a positive sign at a low temperature of $\sim 50 \mathrm{~K}$, consistent with previous observation at the doping level $x=0.2^{1,3,4}$. However, as the thickness decreases to $6.8 \mathrm{~nm}$, the $R_{\mathrm{H}}$ sign-change temperature decreases to $22 \mathrm{~K}$. The $R_{\mathrm{H}}$ even remains negative at the whole temperature range below $300 \mathrm{~K}$ for the films with thickness lower than $6.8 \mathrm{~nm}$. Figure 2(e) presents the thickness dependence of 
the $R_{\mathrm{H}}$ at $20 \mathrm{~K}$ and $300 \mathrm{~K}$, clearly showing a sign-change from positive to negative with decreasing thickness. This suggests a change of the multiband structures upon reducing thickness.

Figure 2(c) shows the temperature dependence of magnetization ( $M$ - $T$ curves) under zero-field cooling (ZFC) mode for $\mathrm{Nd}_{0.8} \mathrm{Sr}_{\mathrm{O} .2} \mathrm{NiO}_{2}$ thin films with thickness varying from 6.1 to $17 \mathrm{~nm}$. The normal-state magnetization shows temperature independence. Upon cooling down, the magnetization drops steeply to negative values, confirming the occurrence of superconducting transition in the thin films. Figure 2(d) shows the zoomed-in $M$ - $T$ curves from 20 down to $2.1 \mathrm{~K}$ with $\mathrm{ZFC}$ and field cooling (FC) for a representative sample with a thickness of $8.4 \mathrm{~nm}$. Similar to the behavior of typical bulk superconductors, a clear diamagnetic transition is seen, and the onset transitions are the same under ZFC and FC modes, unambiguously confirming the existence of the superconducting phase. The ZFC and FC $M$-T curves for other thin films are shown in Supplementary Data Fig. S2. The $T_{\mathrm{c}}$, dia, defined as the onset transition temperature in diamagnetic response, is shown in Fig. 2(f). It is found that $T_{\mathrm{c}}$,dia is slightly lower

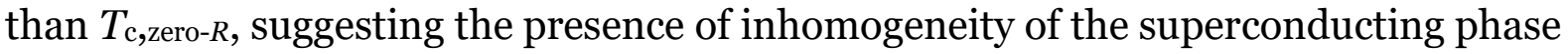
in the thin films. Only well below the zero-resistance temperature, the phase coherence occurs in the entire films, and therefore, the Meissner effect is observed.

The unoccupied states of energy bands are crucial to determine the transport properties and could be detected by X-ray absorption spectroscopy (XAS) on oxygen and transition metal edges. To carefully characterize the electronic structures of samples with pure perovskite phase and the resultant infinite-layer phase, we restrict our XAS measurements on thin films with thickness no more than $11.3 \mathrm{~nm}$, as confirmed by XRD measurements. Figure 3(a) shows the $\mathrm{O} K$ edge XAS of $11.3-\mathrm{nm}$ perovskite $\mathrm{Nd}_{0.8} \mathrm{Sr}_{0.2} \mathrm{NiO}_{3}$ and infinite-layer $\mathrm{Nd}_{0.8} \mathrm{Sr}_{0.2} \mathrm{NiO}_{2}$ thin films. A prominent 
pre-peak at $\sim 528.9 \mathrm{eV}$ is observed near the $\mathrm{O} \mathrm{K}$-edge XAS in $\mathrm{Nd}_{0.8} \mathrm{Sr}_{0.2} \mathrm{NiO}_{3}$ film, which is attributed to the presence of a ligand hole in oxygen ${ }^{18-20}$. In perovskite nickelates, the oxygen $p$ to nickel $d$ band charge-transfer energy is negative, the electrons spontaneously transfer from oxygen ligands to Ni cations, leaving the holes on the oxygen side even without chemical doping ${ }^{18}$. Excitation of oxygen $1 s$ core electrons to such unoccupied states (holes) give rise to the pre-peak in $\mathrm{O} K$-edge XAS. The ligand holes in perovskite $\mathrm{Nd}_{0.8} \mathrm{Sr}_{0.2} \mathrm{NiO}_{3}$ can also be suggested from the $\mathrm{Ni} L_{2,3}$ edge XAS (Fig. 3(b)), in which a shoulder is visible at $\sim 856 \mathrm{eV}$ corresponding to the electron transition from the Ni core-level $2 p$ to $3 d^{8} \underline{L}$ state ( $\underline{L}$ is ligand hole), beside the main sharp peak at $\sim 854.5 \mathrm{eV}$ corresponding to the electron transition from the core-level $2 p$ to $3 d^{7}$ state $^{18}$. As the film is reduced from perovskite to infinite-layer structure, the prominent pre-peak in $\mathrm{Nd}_{0.8} \mathrm{Sr}_{0.2} \mathrm{NiO}_{2}$ film disappears, which is consistent with the previous study in underdoped $R \mathrm{NiO}_{2}(R=\mathrm{La}, \mathrm{Nd})^{19,20}$. Instead, another pre-peak with less intensity at a higher energy of $\sim 530.5 \mathrm{eV}$ is observed, suggesting that the oxygen ligand hole is still present in the infinite-layer film. In the Ni $L_{2,3}$ edge XAS (Fig. 3(b)), the main absorption peaks in both films are observed with a position shift to a lower energy for infinite-layer film, consistent with the reduced $\mathrm{Ni}$ valence state as the structures evolve from perovskite to infinite layer ${ }^{21}$. The shoulder is still observed in the infinite-layer $\mathrm{Nd}_{0.8} \mathrm{Sr}_{0.2} \mathrm{NiO}_{2}$ beside the main peak, even though it shifts to a higher energy position and its intensity is lower compared with that of the perovskite film. This further suggests the presence of oxygen ligand hole state in infinite-layer nickelates.

The parent compound of the canonical cuprate superconductor is a charge-transfer insulator according to the Zaanen-Sawatzky-Allen scheme ${ }^{22}$. The doped holes reside at the oxygen sites due to the strong hybridization of $\mathrm{Cu}-3 d_{x^{2}-y^{2}}$ and $\mathrm{O}-2 p$ orbitals 
forming the $3 d^{9} \underline{L}$ states, and therefore, the pre-peak of $\mathrm{O} K$ edge emerges upon doping ${ }^{23}$. The spin of the doped holes in oxygen sites and spin in $\mathrm{Cu}$ site form the Zhang-Rice singlet state, reducing the cuprate to be an effective single-band system ${ }^{24}$. Whether a similar situation occurs in infinite-layer nickelate, however, is far from clear. The theoretical model suggests that the parent compound $\mathrm{NdNiO}_{2}$ is a Mott-Hubbard insulator, in which the $\mathrm{O} 2 p$ band is below Ni $3 d$ lower Hubbard Band ${ }^{25,26}$. This has been suggested by the EELS and XAS measurements that the pre-peak near the $\mathrm{O} K$ edge is completely suppressed in undoped $\mathrm{NdNiO}_{2}$ and $\mathrm{LaNiO}_{2}{ }^{19,20}$. The pre-peak nature in doped $\mathrm{Nd}_{1-\mathrm{x}} \mathrm{Sr}_{\mathrm{x}} \mathrm{NiO}_{2}$ has not been fully explored by XAS since the STO capping layer obscures the absorption from the underlying nickelate films ${ }^{27}$. Our observation of the obvious pre-peak in $\mathrm{O} K$ edge and shoulder in $\mathrm{Ni} L_{2,3}$ edge in $\mathrm{Nd}_{0.8} \mathrm{Sr}_{0.2} \mathrm{NiO}_{2}$ films, which is similar to the cuprate ${ }^{28}$, suggests the $\mathrm{Ni}-\mathrm{O}$ orbital hybridization in infinite-layer nickelate. Note that the EELS measurements have also shown the pre-peak feature in $\mathrm{Nd}_{1-x} \mathrm{Sr}_{x} \mathrm{NiO}_{2}$ with increasing doping, although the intensity is weak ${ }^{20}$. Moreover, a prominent pre-peak has also been seen in trilayer nickelates $R_{4} \mathrm{Ni}_{3} \mathrm{O}_{8}\left(R=\mathrm{La}\right.$ and $\mathrm{Pr}$ ), which possess the same $\mathrm{NiO}_{2}$ square plane as in the infinite-layer nickelates and an effective 1/3 hole doping ${ }^{21,29}$.

Figures 3(c) and $3(\mathrm{~d})$ show $\mathrm{O} K$ edge and $\mathrm{Ni} L_{2,3}$ edge XAS of infinite-layer $\mathrm{Nd}_{0.8} \mathrm{Sr}_{0.2} \mathrm{NiO}_{2}$ films with different thicknesses. The intensity of pre-peak in $\mathrm{O} K$ edge generally increases with decreasing thickness due to the decreasing occupancy (more empty states), suggesting the increase of $\mathrm{Ni}-\mathrm{O}$ hybridization. The $\mathrm{Ni} L_{2,3}$ peak intensity increases and peak position shifts systematically to higher energies as the thickness decreases. This indicates that with decreasing thickness, the Ni $2 p$ core-level electrons have enhanced binding energy, implying a gradual increase of the oxidation state of Ni. The evolution of XAS may be due to the interface and strain effects with decreasing 
thickness and could be related to the transport properties as shown above. It has been constructed by the bulk electronic structure calculations that the infinite-layer nickelate possesses multiband structures which show the hole pocket originating from a Ni-3 $3 d_{x^{2}-y^{2}}$ orbital and the electron pockets from the rare-earth element $5 d_{x y}$ and $5 d_{3 z^{2}-r^{2}}$ orbitals 2 . Such multiband pictures are consistent with the observed change of $R_{\mathrm{H}}$ in Sr-doped $\mathrm{NdNiO}_{2}$ and $\mathrm{PrNiO}_{2}{ }^{1,3,4,6}$. Our observation is that the $R_{\mathrm{H}}$ of $\mathrm{Nd}_{0.8 \mathrm{Sr}} \mathrm{Sr}_{2} \mathrm{NiO}_{2}$ also changes with the thickness (Fig. 2(b)), possibly related to the alteration of band structure due to the interface and strain effects.

Theoretical calculations suggested the presence of electronic and atomic reconstructions at $\mathrm{NdNiO}_{2} / \mathrm{SrTiO}_{3}$ interfaces and the resultant alteration of band structure at the interface ${ }^{13-16}$. At the surface and interface, the $\mathrm{NiO}_{2}$ layer bends and $\mathrm{Ni}$ is displaced vertically due to atomic reconstruction and/or the presence of residual apical oxygen at the NdO plane. It is expected that as the thickness decreases, the interface and/or strain effects are more pronounced. Indeed, the bending of the $\mathrm{NiO}_{2}$ layer causes the overall increase of the $c$-axis lattice constant, as indicated by the XRD measurement (Fig. 1(b)). The bending of the $\mathrm{NiO}_{2}$ layer and the resultant tilt of $\mathrm{Ni}-\mathrm{O}$ bonding causes the extra extraction of the electron from $\mathrm{Ni}$ to $\mathrm{O}$. This is consistent with our XAS measurement that the oxidation state of $\mathrm{Ni}$ increases as the thickness decreases (Figs. 3(b) and 3(d)). Moreover, it has been revealed that the multiband structures become more pronounced at the interface, for example, the mixture of $d_{x^{2}-y^{2}}$ and $d_{z^{2}}$ states are present near Fermi level at the interface, causing extra electron pockets ${ }^{13-16}$. Therefore, as the thickness decreases, the $R_{\mathrm{H}}$ remains negative below $300 \mathrm{~K}$ (Fig. 2(b)). It was proposed that the pairing state of $\mathrm{Nd}_{1-\mathrm{x}} \mathrm{Sr}_{\mathrm{x}} \mathrm{NiO}_{2}$ changes from $(d+$ is $)$-wave to $d$-wave paring as the doping increases ${ }^{30}$, in line with the crossover of $R_{\mathrm{H}}$ from negative to positive sign 3,4 . Interestingly, the interface/surface effect, which 
caused the negative $R_{\mathrm{H}}$ sign in our result, also induced dominant $s$-wave paring ${ }^{17}$ as opposed to $d$-wave symmetry for the bulk ${ }^{2,31}$. Overall, even though the Meissner effect is confirmed in the nickelate thin films, the interfacial effects due to the atomic reconstruction play an important role in the modification of multiband structures.

\section{Methods}

The perovskite $\mathrm{Nd}_{0.8} \mathrm{Sr}_{0.2} \mathrm{NiO}_{3}$ thin films with different thicknesses were grown on a $\mathrm{TiO}_{2}$-terminated (001) $\mathrm{SrTiO}_{3}$ (STO) substrate using a pulsed laser deposition (PLD) technique with a $248-\mathrm{nm} \mathrm{KrF}$ excimer laser. No capping layer is introduced for all samples. The deposition temperature and oxygen partial pressure $P_{\mathrm{O} 2}$ for all samples were $600{ }^{\circ} \mathrm{C}$ and $150 \mathrm{mTorr}$, respectively. The laser energy density on the target surface was set to be $1.8 \mathrm{Jcm}^{-2}$. After deposition, the samples were annealed for $10 \mathrm{~min}$ at $600{ }^{\circ} \mathrm{C}$ and 150 mTorr and then cooled down to room temperature at a rate of $8{ }^{\circ} \mathrm{C} / \mathrm{min}$. The as-grown samples were cut into pieces with a size of around $2.5 \times 5$ $\mathrm{mm}^{2}$. The pieces were then embedded with about $0.15 \mathrm{~g}$ of $\mathrm{CaH}_{2}$ powder and wrapped in aluminum foil, and then placed into the PLD chamber for reduction. Using the PLD heater, the wrapped samples were heated to $340-360{ }^{\circ} \mathrm{C}$ at a rate of $25^{\circ} \mathrm{C} / \mathrm{min}$ and kept for 80 minutes, and then cooled down to room temperature at a rate of $25^{\circ} \mathrm{C} / \mathrm{min}$. The wire connection for the electrical transport measurement was made by $\mathrm{Al}$ ultrasonic wire bonding. The electrical transport and magnetization measurements were performed using a Quantum Design Physical Property Measurement System and Superconducting Quantum Interference Device Magnetometer, respectively. The Xray diffraction (XRD) measurement was done in the X-ray Diffraction and Development (XDD) beamline at Singapore Synchrotron Light Source (SSLS) with an X-ray wavelength of $\lambda=1.5404 \AA$. The XAS measurements were performed using 
linearly polarized X-ray from the Soft X-ray-ultraviolet (SUV) beamline at SSLS, using a total electron yield (TEY) detection method. The incidence angle $(90-\theta)^{\circ}$ of X-rays refers to the normal of the sample surface, which was varied by rotating the polar angle of the sample. The spectra were measured in a grazing-incident alignment $\left(\theta=20^{\circ}\right)$ to obtain better sample signals. The spectra were normalized to the integrated intensity at the tail of the spectra after subtracting an energy-independent background. The high-angle annular dark-field scanning transmission electron microscopy (HAADF-STEM) imaging was carried out at $200 \mathrm{kV}$ using a JEOL ARM20oF microscope, and the cross-sectional TEM specimens were prepared by a focused ion beam machine (FEI Versa 3D).

\section{Acknowledgments}

This research is supported by the Agency for Science, Technology, and Research (A*STAR) under its Advanced Manufacturing and Engineering (AME) Individual Research Grant (IRG) (A1983co034) and the Singapore National Research Foundation (NRF) under the Competitive Research Programs (CRP Grant No. NRFCRP15-2015-01). P. Y. is supported by Singapore Synchrotron Light Source (SSLS) via NUS Core Support C-380-003-003-001. The authors would also like to acknowledge the SSLS for providing the facility necessary for conducting the research. The Laboratory is a National Research Infrastructure under the National Research Foundation (NRF) Singapore. 


\section{Author Contributions}

SWZ and AA conceived the project. SWZ prepared the thin films and conducted the electrical and magnetic measurements with the assistance from $\mathrm{KH}, \mathrm{ZH}, \mathrm{YC}, \mathrm{LEC}$, DYW, ZTZ and ZSL. SWZ, PY, CST and XMY conducted the XRD measurements. XMY, CZD, CST and ATSW conducted the XAS measurements. CJL and SJP conducted the STEM measurements. SWZ and AA wrote the manuscript with contributions from all authors. All authors have discussed the results and the interpretations.

\section{References}

1. D. Li, K. Lee, B. Y. Wang, M. Osada, S. Crossley, H. R. Lee, Y. Cui, Y. Hikita, H. Y. Hwang, Superconductivity in an infinite-layer nickelate, Nature, 572, 624-627 (2019).

2. A. S. Botana, F. Bernardini, A. Cano, Nickelate superconductors: an ongoing dialog between theory and experiments, https://arxiv.org/abs/2012.02764, (2020).

3. D. Li, B.Y. Wang, K. Lee, S. P. Harvey, M. Osada, B. H. Goodge, L. F. Kourkoutis, H. Y. Hwang, Superconducting dome in $\mathrm{Nd}_{1-x} \mathrm{Sr}_{\mathrm{x}} \mathrm{NiO}_{2}$ infinite layer films, Phys. Rev. Lett., 125, 027001 (2020).

4. S. Zeng, C. S. Tang, X. Yin, C. Li, M. Li, Z. Huang, J. Hu, W. Liu, G.J. Omar, H. Jani, Z. S. Lim, K. Han, D. Wan, P. Yang, S. J. Pennycook, A. T. S. Wee, A. Ariando, Phase diagram and superconducting dome of infinite-layer $\mathrm{Nd}_{1-\mathrm{x}} \mathrm{Sr}_{\mathrm{x}} \mathrm{NiO}_{2}$ thin films, Phys. Rev. Lett., 125, 147003 (2020).

5. Q. Gu, Y. Li, S. Wan, H. Li, W. Guo, H. Yang, Q. Li, X. Zhu, X. Pan, Y. Nie, H.-H. Wen, Single-particle tunneling spectrum of superconducting $\mathrm{Nd}_{1-\mathrm{x}} \mathrm{Sr}_{\mathrm{x}} \mathrm{NiO}_{2}$ thin films, Nature Commun., 11, 6027 (2020).

6. M. Osada, B.Y. Wang, K. Lee, D. Li, H. Y. Hwang, Phase diagram of infinite layer praseodymium nickelate $\operatorname{Pr}_{1-x} \mathrm{Sr}_{x} \mathrm{NiO}_{2}$ thin films, Phys. Rev. Mater., 4, 121801 (2020). 
7. B. Y. Wang, D. Li, B. H. Goodge, K. Lee, M. Osada, S. P. Harvey, L. F. Kourkoutis, M. R. Beasley, H. Y. Hwang, Isotropic Pauli-limited superconductivity in the infinite-layer nickelate $\mathrm{Nd}_{0.775} \mathrm{Sr}_{0.225} \mathrm{NiO}_{2}$, Nat. Phys., 17, 473-477 (2021).

8. Q. Gao, Y. Zhao, X. Zhou, Z. Zhu, Preparation of superconducting thin film of infinite-layer nickelate $\mathrm{Nd}_{0.8} \mathrm{Sr}_{0.2} \mathrm{NiO}_{2}$, https://arxiv.org/abs/2102.10292, (2021).

9. X. Zhou, Z. Feng, P. Qin, H. Yan, X. Wang, P. Nie, H. Wu, X. Zhang, H. Chen, Z. Meng, Z. Zhu, Z. Liu, Negligible oxygen vacancies, low critical current density, electric-field modulation, in-plane anisotropic and high-field transport of a superconducting $\quad \mathrm{Nd}_{0.8} \mathrm{Sr}_{0.2} \mathrm{NiO}_{2} / \mathrm{SrTiO}_{3} \quad$ heterostructure, https://arxiv.org/abs/2104.07316, (2021).

10. Q. Li, C. He, J. Si, X. Zhu, Y. Zhang, H. H. Wen, Absence of superconductivity in bulk $\mathrm{Nd}_{1-\mathrm{x}} \mathrm{Sr}_{\mathrm{x}} \mathrm{NiO}_{2}$, Commun. Mater., 1, 16 (2020).

11. B. X. Wang, H. Zheng, E. Krivyakina, O. Chmaissem, P. P. Lopes, J. Lynn, L. C. Gallington, Y. Ren, S. Rosenkranz, J. Mitchell, D. Phelan, Synthesis and characterization of bulk $\mathrm{Nd}_{1-x} \mathrm{Sr}_{x} \mathrm{NiO}_{2}$ and $\mathrm{Nd}_{1-\mathrm{x}} \mathrm{Sr}_{\mathrm{x}} \mathrm{NiO}_{3}$, Phys. Rev. Mater., 4, 084409 (2020).

12. C. He, X. Ming, Q. Li, X. Zhu, J. Si, H. H. Wen, Synthesis and physical properties of perovskite $\mathrm{Sm}_{1-\mathrm{x}} \mathrm{Sr}_{\mathrm{x}} \mathrm{NiO}_{3}(\mathrm{x}=0,0.2)$ and infinite-layer $\mathrm{Sm}_{0.8} \mathrm{Sr}_{0.2} \mathrm{NiO}_{2}$ nickelates, J. Phys. Condens. Matter, DOI 10.1088/1361-648X/abfb9o (2021).

13. B. Geisler, R. Pentcheva, Fundamental difference in the electronic reconstruction of infinite-layer versus perovskite neodymium nickelate films on $\mathrm{SrTiO}_{3}$ (001), Phys. Rev. B, 102, 020502 (2020).

14. R. He, P. Jiang, Y. Lu, Y. Song, M. Chen, M. Jin, L. Shui, Z. Zhong, Polarity-induced electronic and atomic reconstruction at $\mathrm{NdNiO}_{2} / \mathrm{SrTiO}_{3}$ interfaces, Phy. Rev. B, 102, 035118 (2020).

15. F. Bernardini, A. Cano, Stability and electronic properties of $\mathrm{LaNiO}_{2} / \mathrm{SrTiO}_{3}$ heterostructures, J. Phys. Mater., 3, 03LTo1 (2020).

16. Y. Zhang, L. F. Lin, W. Hu, A. Moreo, S. Dong, E. Dagotto, Similarities and differences between nickelate and cuprate films grown on a $\mathrm{SrTiO}_{3}$ substrate, Phys. Rev. B, 102, 195117 (2020). 
17. X. Wu, K. Jiang, D. Di Sante, W. Hanke, A. Schnyder, J. Hu, R. Thomale, Surface $s$-wave superconductivity for oxide-terminated infinite-layer nickelates, https://arxiv.org/abs/2008.06009, (2020).

18. V. Bisogni, S. Catalano, R.J. Green, M. Gibert, R. Scherwitzl, Y. Huang, V. N. Strocov, P. Zubko, S. Balandeh, J. M. Triscone, Ground-state oxygen holes and the metal-insulator transition in the negative charge-transfer rare-earth nickelates, Nat. Commun., 7, 13017 (2016).

19. M. Hepting, D. Li, C. J. Jia, H. Lu, E. Paris, Y. Tseng, X. Feng, M. Osada, E. Been, Y. Hikita, Y. D. Chuang, Z. Hussain, K. J. Zhou, A. Nag, M. Garcia-Fernandez, M. Rossi, H. Y. Huang, D. J. Huang, Z. X. Shen, T. Schmitt, H. Y. Hwang, B. Moritz, J. Zaanen, T. P. Devereaux, W. S. Lee, Electronic structure of the parent compound of superconducting infinite-layer nickelates, Nat. Mater., 19, 381-385 (2020).

20. B. H. Goodge, D. Li, K. Lee, M. Osada, B. Y. Wang, G. A. Sawatzky, H. Y. Hwang, L. F. Kourkoutis, Doping evolution of the Mott-Hubbard landscape in infinitelayer nickelates, Proc.Natl. Acad. Sci. U. S. A., 118, e2007683118 (2021).

21. J. Zhang, A. Botana, J. Freeland, D. Phelan, H. Zheng, V. Pardo, M. Norman, J. Mitchell, Large orbital polarization in a metallic square-planar nickelate, Nat. Phys., 13, 864-869 (2017).

22. J. Zaanen, G. Sawatzky, J. Allen, Band gaps and electronic structure of transitionmetal compounds, Phys. Rev. Lett., 55, 418 (1985).

23. C. Chen, F. Sette, Y. Ma, M. Hybertsen, E. Stechel, W. Foulkes, M. Schulter, S. W. Cheong, A. Cooper, L. Rupp Jr, Electronic states in $\mathrm{La}_{2-\mathrm{x}} \mathrm{Sr}_{\mathrm{x}} \mathrm{CuO}_{4+\delta}$ probed by soft x-ray absorption, Phys. Rev. Lett., 66, 104 (1991).

24. F. C. Zhang, T. M. Rice, Effective Hamiltonian For The Superconducting Cu Oxides, Phys. Rev. B, 37, 3759-3761 (1988).

25. K. W. Lee, W. Pickett, Infinite-layer $\mathrm{LaNiO}_{2}$ : $\mathrm{Ni}^{1+}$ is not $\mathrm{Cu}^{2+}$, Phys. Rev. B, 70, 165109 (2004).

26. M. Jiang, M. Berciu, G. A. Sawatzky, Critical Nature of the Ni Spin State in Doped $\mathrm{NdNiO}_{2}$, Phys. Rev. Lett., 124, 207004 (2020).

27. M. Rossi, H. Lu, A. Nag, D. Li, M. Osada, K. Lee, B. Y. Wang, S. Agrestini, M. Garcia-Fernandez, Y. D. Chuang, Z. X. Shen, H. Y. Hwang, B. Moritz, K. J. Zhou, 
T. P. Devereaux, W. S. Lee, Orbital and Spin Character of Doped Carriers in Infinite-Layer Nickelates, https://arxiv.org/abs/2011.00595, (2020).

28. D. Meyers, S. Mukherjee, J.-G. Cheng, S. Middey, J.-S. Zhou, J. Goodenough, B. Gray, J. Freeland, T. Saha-Dasgupta, J. Chakhalian, Zhang-Rice physics and anomalous copper states in A-site ordered perovskites, Sci. Rep., 3, 1834 (2013).

29. J. Q. Lin, P. Villar Arribi, G. Fabbris, A. S. Botana, D. Meyers, H. Miao, Y. Shen, D. G. Mazzone, J. Feng, S. G. Chiuzbaian, A. Nag, A. C. Walters, M. Garcia-Fernandez, K. J. Zhou, J. Pelliciari, I. Jarrige, J. W. Freeland, J. Zhang, J. F. Mitchell, V. Bisogni, X. Liu, M. R. Norman, M. P. M. Dean, Strong Superexchange in a $d^{9-\delta}$ Nickelate Revealed by Resonant Inelastic X-Ray Scattering, Phys. Rev. Lett., 126, 087001 (2021).

30. Z. Wang, G. M. Zhang, Y. F. Yang, F. C. Zhang, Distinct pairing symmetries of superconductivity in infinite-layer nickelates, Phys. Rev. B, 102, 220501 (2020).

31. X. Wu, D. Di Sante, T. Schwemmer, W. Hanke, H. Y. Hwang, S. Raghu, R. Thomale, Robust $d_{\mathrm{x} 2-\mathrm{y} 2}$-wave superconductivity of infinite-layer nickelates, Phys. Rev. B, 101, 060504(R) (2020). 


\section{Figures and Captions:}
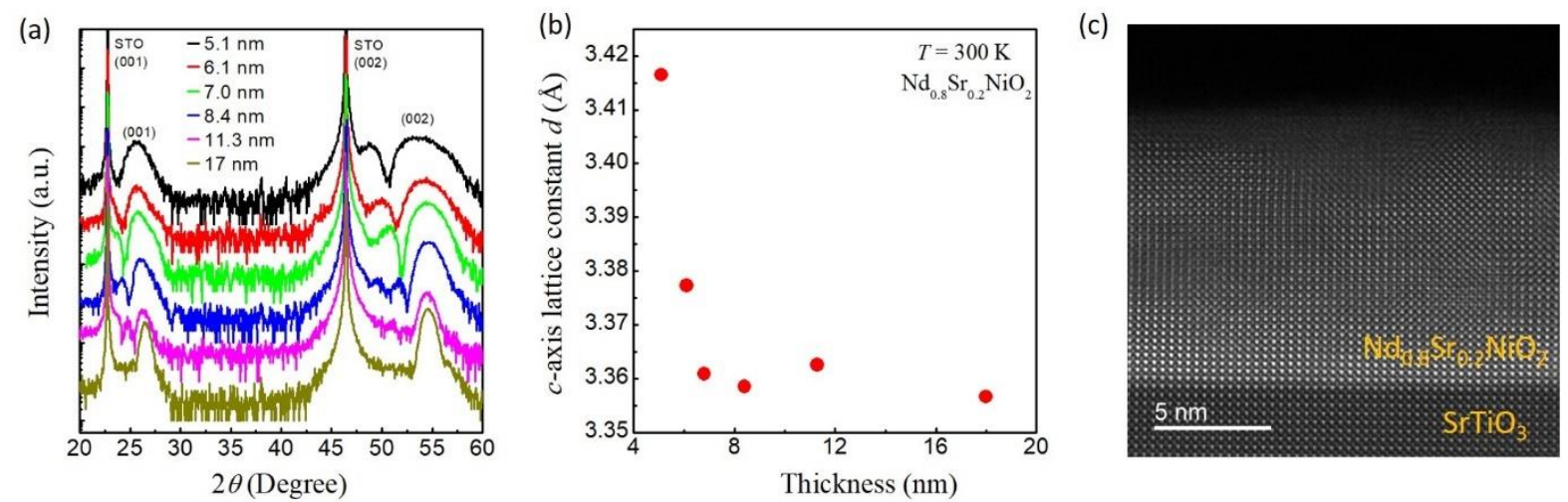

Figure 1: Thickness dependence of the infinite layer structure. (a) The XRD $\theta-2 \theta$ scan patterns of the $\mathrm{Nd}_{0.8} \mathrm{Sr}_{0.2} \mathrm{NiO}_{2}$ thin films with different thicknesses on $\mathrm{SrTiO}_{3}$ substrates. The intensity is vertically displaced for clarity. (b) The room-temperature $c$-axis lattice constants, $d$, as a function of thickness, as calculated from the (oo1) peak positions. (c) The HAADF-STEM image of the $11.3-\mathrm{nm} \mathrm{Nd}_{0.8} \mathrm{Sr}_{0.2} \mathrm{NiO}_{2}$ on $\mathrm{SrTiO}_{3}$ substrate. 

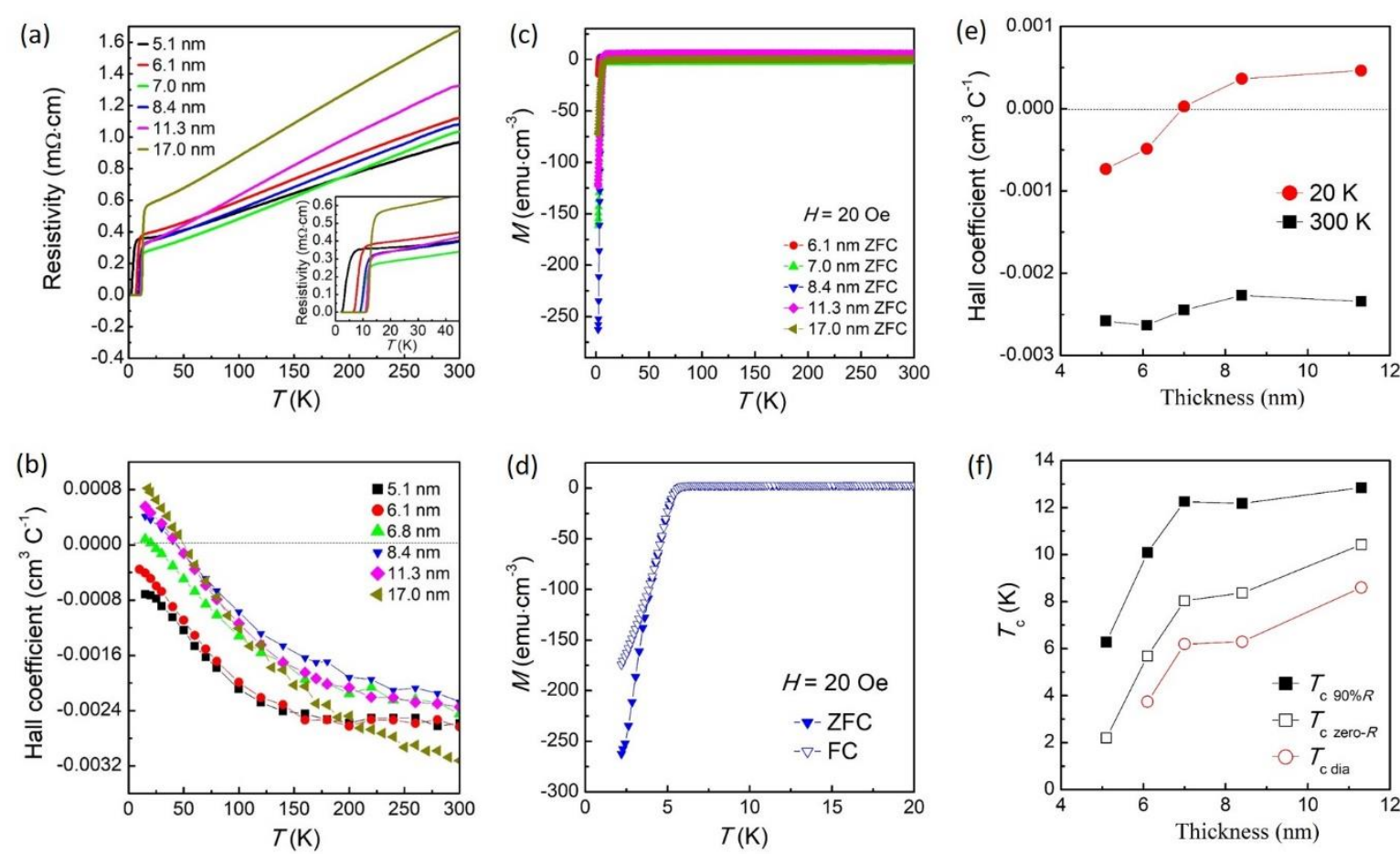

\section{Figure 2: Thickness dependence of the transition temperature, diamagnetic} response, and Hall coefficient. (a) The resistivity versus temperature $(\rho-T)$ curves of the $\mathrm{Nd}_{0.8} \mathrm{Sr}_{0.2} \mathrm{NiO}_{2}$ thin films with different thicknesses from 5.1 to $17.0 \mathrm{~nm}$. The inset shows the zoomed-in $\rho$-T curves at temperatures from 50 to $2 \mathrm{~K}$. (b) The temperature dependence of the normal-state Hall coefficients $R_{\mathrm{H}}$. (c) The temperature dependence of magnetization ( $M-T$ curve) with zero-field cooling (ZFC) for $\mathrm{Nd}_{0.8} \mathrm{Sr}_{0.2} \mathrm{NiO}_{2}$ thin films with different thicknesses from 6.1 to $17.0 \mathrm{~nm}$. (d) The zoomed-in $M$ - $T$ curves at temperatures from 20 to $2.1 \mathrm{~K}$ with field cooling (FC) and ZFC for the sample with a thickness of $8.4 \mathrm{~nm}$. The measurement and cooling fields are 20 Oe. The magnetic field is applied perpendicularly to the $a-b$ plane. (e) The $R_{\mathrm{H}}$ at $T=300$ and $20 \mathrm{~K}$ as a function of thickness. (f) The critical temperature, $T_{\mathrm{c}}$, as a function of thickness. The $T_{\mathrm{c}, 90 \% R}$ is defined as the temperature at which the resistivity drops to $90 \%$ of

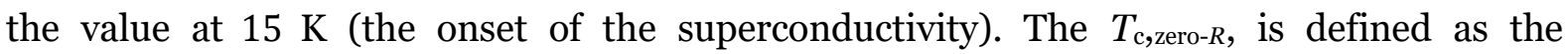
temperature at which the resistivity drops to be zero and $T_{\mathrm{c} \text {,dia }}$ is defined as the temperature at the onset of the diamagnetic response. The dash lines in (b) and (e) show the position where the $R_{\mathrm{H}}$ is zero. 

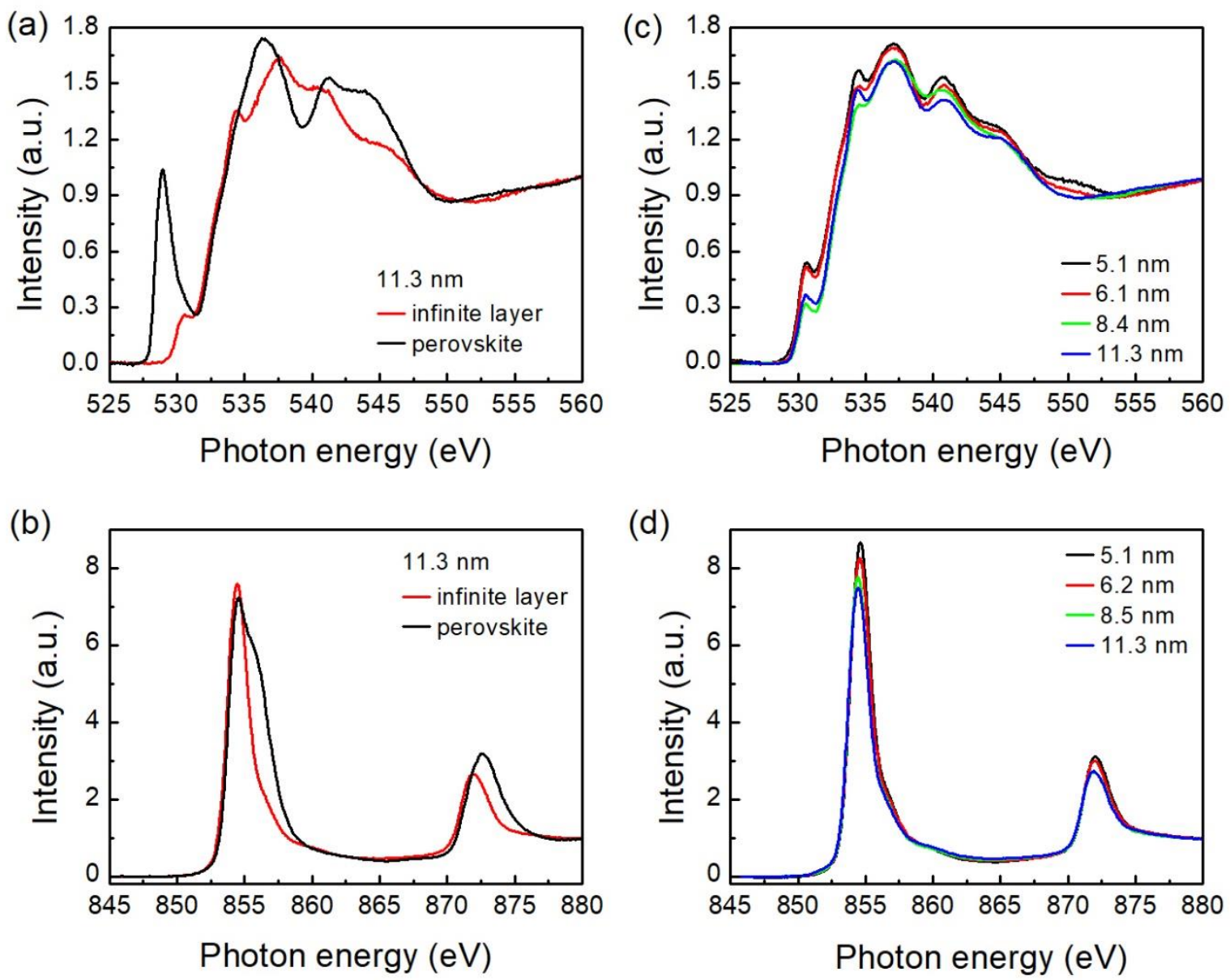

Figure 3: Thickness dependence of the X-ray Absorption Spectroscopy. (a) The O $K$ edge and (b) Ni $L_{2,3}$ edge XAS of $11.3-\mathrm{nm}$ perovskite $\mathrm{Nd}_{0.8} \mathrm{Sr}_{0.2} \mathrm{NiO}_{3}$ and infinite-layer $\mathrm{Nd}_{0.8} \mathrm{Sr}_{0.2} \mathrm{NiO}_{2}$ thin films. (c) The $\mathrm{O} K$ and (d) $\mathrm{Ni} L_{2,3}$ edge of infinite-layer $\mathrm{Nd}_{0.8} \mathrm{Sr}_{0.2} \mathrm{NiO}_{2}$ films with different thicknesses. 


\section{Supplementary Data}
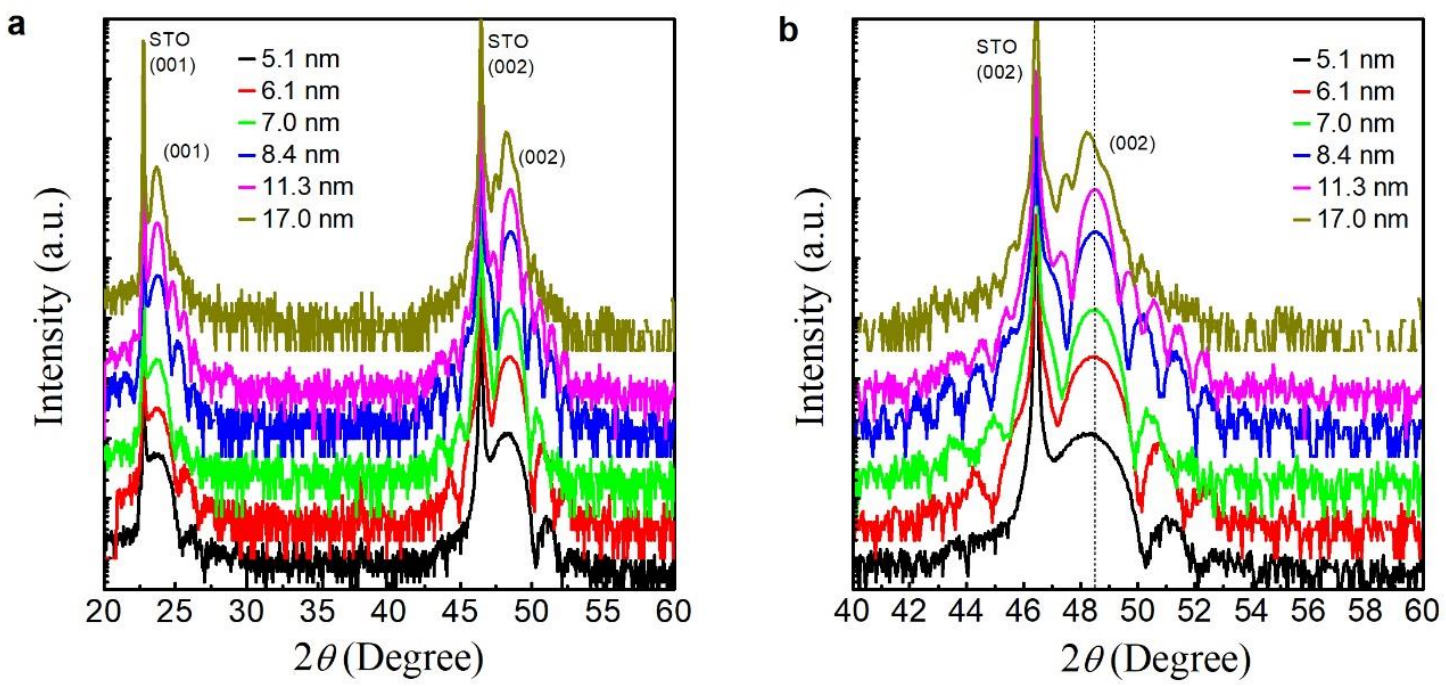

Supplementary Figure S1: (a) The XRD $\theta-2 \theta$ scan patterns of the as-grown $\mathrm{Nd}_{0.8} \mathrm{Sr}_{0.2} \mathrm{NiO}_{3}$ thin films with different thicknesses on STO substrates. The intensity is vertically displaced for clarity. Only the $(00 l)$ perovskite peaks are observed, where $l$ is an integer, confirming the $c$-axis oriented epitaxial growth. (b) The zoomed-in XRD $\theta-2 \theta$ scan patterns at angles from 40 to 60 degrees. The black dash line shows the position of the (002) perovskite peaks for thin films with different thicknesses. One can see that for thin films with thicknesses from 5.1 to $11.3 \mathrm{~nm}$, the (002) peaks are at the same position, indicating the same $c$-lattice constant. For the film with a thickness of $17 \mathrm{~nm}$, the presence of multiple peaks is seen at (002) position, suggesting the presence of mixed phases as the film thickness is increased, consistent with previous reports. The thickness is calculated through the period of thickness oscillations in the vicinity of the (002) peak. 
a
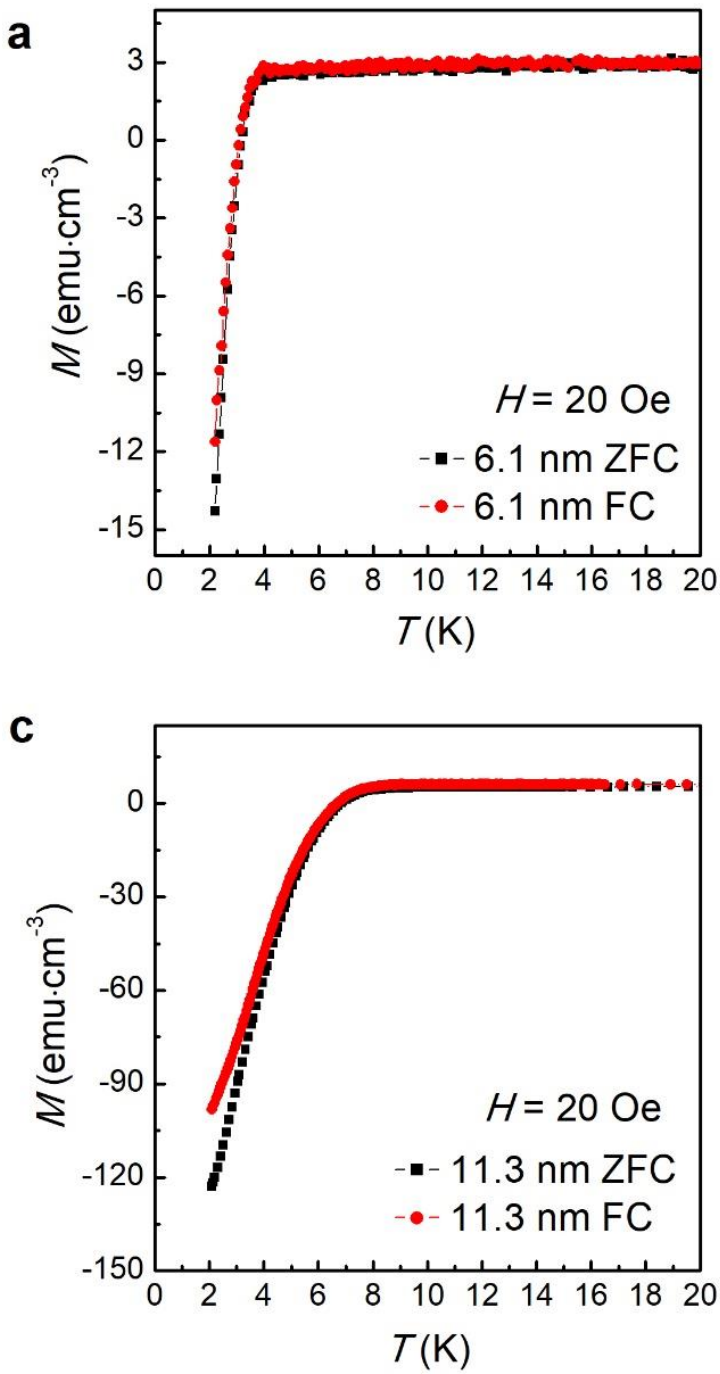
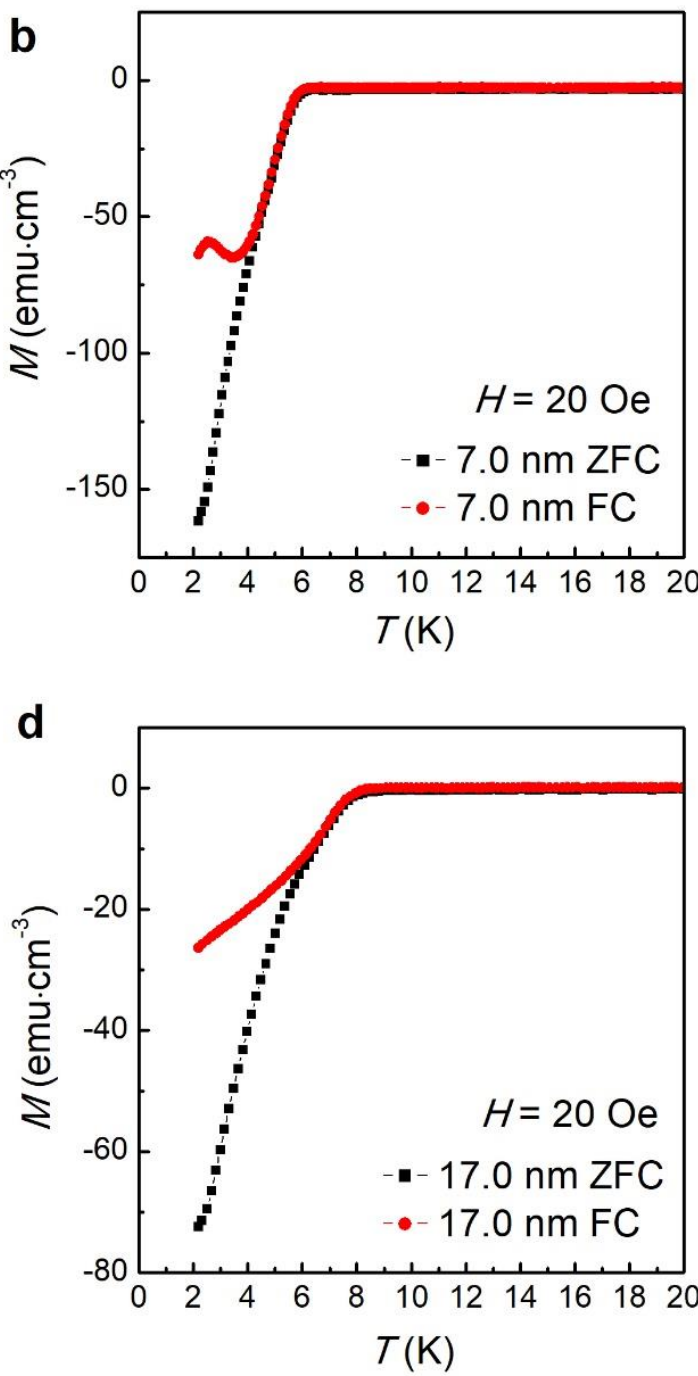

Supplementary Figure S2: Temperature dependence of magnetization ( $M$ - $T$ curve) with zero-field cooling (ZFC) and field cooling (FC) for $\mathrm{Nd}_{0.8} \mathrm{Sr}_{0.2} \mathrm{NiO}_{2}$ thin films with different thickness of (a) $6.1 \mathrm{~nm}$, (b) $7 \mathrm{~nm}$, (c) $11.3 \mathrm{~nm}$ and (d) $17 \mathrm{~nm}$. The $M-T$ curves are shown at temperatures from 20 to $2.1 \mathrm{~K}$. The measurement and cooling fields are 20 Oe. The magnetic field is applied perpendicularly to the $a-b$ plane. 